\title{
Diagnóstico precoce e o tratamento cirúrgico do polegar em gatilho congênito na criança utilizando o sistema de internação hospital-dia
}

\author{
Early diagnosis and surgical treatment of congenital trigger thumb in children using \\ the day hospital admission system
}

Walter Yoshinori Fukushima', Edison Noboru Fujiki', Gustavo Mantovani Ruggiero', Álvaro Baik Cho', Márcio Aurélio Aita', Fabiano Prata Nascimento' ${ }^{2}$ Thiago da Motta Mattar', Carlo Milani'

\begin{abstract}
Resumo
Introdução: o polegar em gatilho congênito ou tenossinovite estenosante do polegar é a dificuldade de extensão do polegar, identificada nos primeiros meses de vida. Objetivo: a proposta deste estudo foi discutir o tratamento cirúrgico com os resultados, as complicações e as vantagens na utilização da internação hospital-dia. Método: no período de fevereiro de 2001 até janeiro de 2008, verificaram-se 25 crianças entre 3 meses e 6 anos, portadoras de polegar em gatilho congênito. Foram operadas 35 mãos, e 10 casos eram bilaterais. Observamos que a manifestação clínica quanto à dificuldade de extensão do polegar estava presente em todas as mãos operadas e nenhuma criança queixava-se de dor. As crianças foram submetidas à mesma técnica cirúrgica, anestesia geral inalatória, com incisão transversa na prega volar metacarpofalangeana do polegar acometido e abertura longitudinal da polia flexora A1. Não foi utilizado antibiótico e as crianças foram internadas pelo sistema hospital-dia. Resultados: Quinze crianças eram do sexo masculino e dez do sexo feminino. Dos pacientes com lesão bilateral, sete eram do sexo masculino e três do feminino. Quanto ao lado acometido, obtivemos sete polegares direitos, oito esquerdos e dez bilaterais. Como complicações, não houve nenhuma recidiva; um polegar evoluiu com infecção cutânea superficial e dois polegares apresentaram deiscência parcial da sutura após a retirada dos pontos. Conclusões: o polegar em gatilho congênito ou tenossinovite estenosante do polegar na criança pode ser tratado com segurança através da abertura simples da polia flexora A1, utilizando-se a internação hospital-dia.
\end{abstract}

Palavras-chave: Dedo em gatilho; encarceramento do tendão; tenossinovite; polegar.

\section{Abstract}

Introduction: The congenital trigger disorder or stenosing tenosynovitis of the thumb is the difficulty of extending the thumb, identified in early months of life. Objective: The purpose this study was to discuss the operative results, complications and advantages of the day hospital system. Method: This study was carried out from February 2001 to January 2008, with 25 children aging from 3 months to 6 years old with congenital trigger finger thumb. Thirty-five hands were submitted to surgery, and ten children had both hands attacked. The limited extension of the thumb was the clinical characteristic observed in all operated hands and no child had pain complaint. All children were submitted to the same surgical technique, by the use of inhalational general anesthetics, with transversal incision in the metacarpophalangeal volar pleat of the thumb and longitudinal resection of the flexor pulley A1. Antibiotic therapy was not used and all children were admitted by the day hospital system. Results: fifteen were boys and ten were girls. Seven boys and three girls had the pathology in both hands. Comparing the incidence of each side, we have found seven right congenital trigger thumbs, eight left and ten occurring in both sides. The complications found were: one thumb had superficial cutaneous infection and two thumbs presented dehiscence of the incision after the removal of the suture. There were no cases of recurrence of the disease after surgery. Conclusion: we concluded that congenital trigger disorder or stenosing tenosynovitis in children can be safely treated with simple incision of flexor pulley A1 and with day hospital system.

Keywords: Trigger finger disorder; tendon entrapment; tenosynovitis; thumb.

Recebido: $19 / 5 / 2009$

Revisado: $26 / 9 / 2009$

Aprovado: 28/12/2009

Trabalho realizado no Hospital Estadual Mário Covas da Fundação do ABC, Santo André (SP), Brasil.

Disciplina de Ortopedia e Traumatologia da Faculdade de Medicina do ABC (FMABC), Santo André (SP), Brasil

2 Hospital Estadual Mário Covas da Fundação do ABC, Santo André (SP), Brasil

Endereço para correspondência: Walter Yoshinori Fukushima - Rua das Aroeiras, 191 - Jardim - CEP 09090-000 - Santo André (SP) -

E-mail:walterfukushima@ig.com.br 


\section{Introdução}

As crianças, após o nascimento, são submetidas a vários testes clínicos para deteç̧ão de doenças e alterações congênitas do sistema musculoesquelético. Porém, na maioria das vezes, não é detectada a dificuldade de extensão do polegar. O quadro clínico não é doloroso, sendo diferente do dedo em gatilho no adulto. Com o crescimento da mão e do polegar, o dedo vai adquirindo a atitude em flexão, ou seja, os pais notam que a criança tem dificuldade para estender o polegar, não fazendo o sinal de positivo.

O polegar em gatilho congênito ou tenossinovite estenosante do polegar, quando detectado nas fases iniciais da vida, pode ter boa evolução, com orientação e exercícios passivos de extensão'. Porém, quando o diagnóstico ocorre tardiamente ou não responde ao tratamento clínico, indica-se o tratamento operatório. Assim, o objetivo deste trabalho é discutir o diagnóstico precoce e o tratamento cirúrgico com os resultados, as complicações e as vantagens da utilização da internação hospital-dia.

\section{Método}

Durante o período de fevereiro de 2001 a janeiro de 2008, foram operadas 25 crianças entre um e seis anos de idade, portadoras de polegar em gatilho congênito ou tenossinovite estenosante do polegar. Foram operados 35 polegares, sendo 10 pacientes com casos bilaterais; 15 crianças operadas eram do sexo masculino e 10 do sexo feminino. Dos pacientes com lesão bilateral, 7 eram do sexo masculino e 3 do sexo feminino. Quanto ao lado acometido, houve 17 polegares direitos e 18 esquerdos. Quanto à etnia, 15 crianças eram brancas e 10 não brancas (Tabela I).

Os critérios de inclusão foram: pacientes até 17 anos que apresentavam dificuldade à extensão ativa da interfalange distal do polegar com bloqueio em flexão e que não sentiam dor. Foram excluídos pacientes com outros dedos acometidos e que apresentavam doenças sistêmicas associadas. O procedimento cirúrgico foi igual para todos os pacientes, com anestesia geral inalatória, uso de torniquete (faixa de Esmarch) na raiz do membro superior, incisão transversa no polegar com cerca de $5 \mathrm{~mm}$ na prega volar metacarpofalangeana, identificação e abertura longitudinal da polia flexora Al. Foi feita exploração cirúrgica em todos os casos, tanto local como proximal, e na porção imediatamente distal do tendão quanto a possível existência de cistos, tumores e outras alterações. A pele foi suturada com fio cirúrgico absorvível poliglecaprone 25 (Monocryl $\left.{ }^{\circledR}\right)$ 5-0. Após o curativo, as mãos foram submetidas ao enfaixamento compressivo, mantendo o polegar em abdução e extensão. Não utilizamos antibiótico nem imobilização gessada, e as crianças foram operadas pelo sistema de internação hospital-dia. Esta pesquisa foi aprovada pelo Comitê de Ética da Faculdade de Medicina do ABC (FMABC), sob o parecer 128/2009.

\section{Resultados}

O tempo de uso do torniquete foi de, no mínimo, 18 minutos e, no máximo, de 36 minutos. Não tivemos nenhuma complicação anestésica; em 3 crianças foram aplicados, no local, $2 \mathrm{~mL}$ de cloridrato de bupivacaína $0,5 \%$, sem vasoconstrictor, por solicitação dos anestesistas. Todos apresentavam boa mobilidade do polegar, com extensão ativa já no primeiro retorno ambulatorial, não necessitando de fisioterapia assistida, apenas da estimulação materna no período pós-operatório. Não houve nenhuma recidiva, e como complicações obtivemos um polegar com infecção cutânea superficial e dois polegares com deiscência parcial da sutura após a retirada dos pontos, que evoluíram para cura sem intercorrências.

\section{Discussão}

Conceitualmente, é possível discutir se o polegar em gatilho congênito é uma doença congênita ou adquirida, pois não se encaixa em nenhum grupo na classificação clássica das deformidades congênitas da mão ${ }^{2}$. Por manifestar-se próximo ao período natal, poderia ser chamada de congênita; além disso, não tem causa traumática ou inflamatória que justifique a dificuldade ou impossibilidade de extensão do polegar para ser chamada de adquirida. O diagnóstico é eminentemente clínico, devendo ser realizado ou no momento do exame neonatal ou pelo pediatra nos retornos ambulatoriais, observando-se a posição em flexão da falange distal do polegar e a dificuldade de extensão da mesma. A radiografia deve ser realizada para descartar problemas osteoarticulares, dentre eles, o polegar curto congênito. Em nossa casuística, a maioria dos casos foi notada pela primeira vez pelos familiares, que observaram a posição do polegar e a impossibilidade de realizar o sinal de positivo.

O termo dedo em gatilho congênito tem a mesma conotação clínica do dedo em gatilho do adulto por apresentar flexão do dedo e dificuldade para extensão. Entretanto, no adulto ocorre bloqueio tanto em flexão quanto em extensão e, quando ocorre o desbloqueio, existe dor $\mathrm{Na}$ criança, ao contrário, não ocorre o bloqueio em extensão e o que se observa é a dificuldade em estender a falange distal sem que ocorra ressalto, podendo surgir dor quando se força exageradamente a extensão passiva. Em geral no adulto acomete a terceira e quarta décadas, consequentes a uma tenossinovite traumática, atividades repetitivas, doenças

Tabela I - Número total de pacientes, número de pacientes quanto ao sexo, número de polegares quanto ao lado acometido e epidemiologia quanto à etnia.

\begin{tabular}{lcccc}
\hline Número total de Pacientes & 25 & & & \\
Sexo & $\begin{array}{c}15 \\
\text { meninos }\end{array}$ & $\begin{array}{c}10 \\
\text { meninas }\end{array}$ & & \\
$\begin{array}{l}\text { Número de polegares } \\
\text { quanto ao lado acometido }\end{array}$ & $\begin{array}{c}17 \\
\text { (polegar } \\
\text { direito) }\end{array}$ & $\begin{array}{c}18 \\
\text { (polegar } \\
\text { esquerdo) }\end{array}$ & $\begin{array}{c}10 \\
\text { (bilateral) }\end{array}$ & $\begin{array}{c}\text { Total de 35 } \\
\text { polegares }\end{array}$ \\
Etnia & $\begin{array}{c}15 \\
\text { brancos }\end{array}$ & $\begin{array}{c}10 \\
\text { não-brancos }\end{array}$ \\
\end{tabular}


associadas, podendo atingir outros dedos, o que não se observa na forma congênita ${ }^{3}$. Portanto, a nomenclatura dedo em gatilho congênito pode não ser o termo mais adequado para esta afecção. Durante o ato cirúrgico, observamos o espessamento da polia flexora A1 (Figura 1) e espessamento do tendão flexor do polegar, observado, também, no adulto.

Estudos histopatológicos ${ }^{4}$ associaram o dedo em gatilho congênito a metaplasia fibrocartilaginosa da camada interna da polia A1, e não a processo inflamatório da membrana sinovial, descaracterizando o termo tenossinovite. Diversas condições podem relacionar-se ao dedo em gatilho, entre elas, artrite reumatoide, diabetes, gota, pacientes submetidos a hemodiálise, tumores do tendão ou de sua bainha, configurando os casos de dedos em gatilhos secundários. Não observamos nas crianças nenhuma relação com doenças ou causas secundárias.

O tratamento clínico com infiltração local de corticosteroide ${ }^{5-7}$ pode ser realizado. $\mathrm{Na}$ nossa casuística não foi realizada a infiltração local em nenhum polegar. A fisioterapia deve ser realizada nos pacientes adultos. Algumas crianças, provenientes de outros serviços, haviam feito esse tratamento, porém sem sucesso. Em nossos casos, não orientamos fisioterapia ou manipulações caseiras, pois os pacientes se apre-

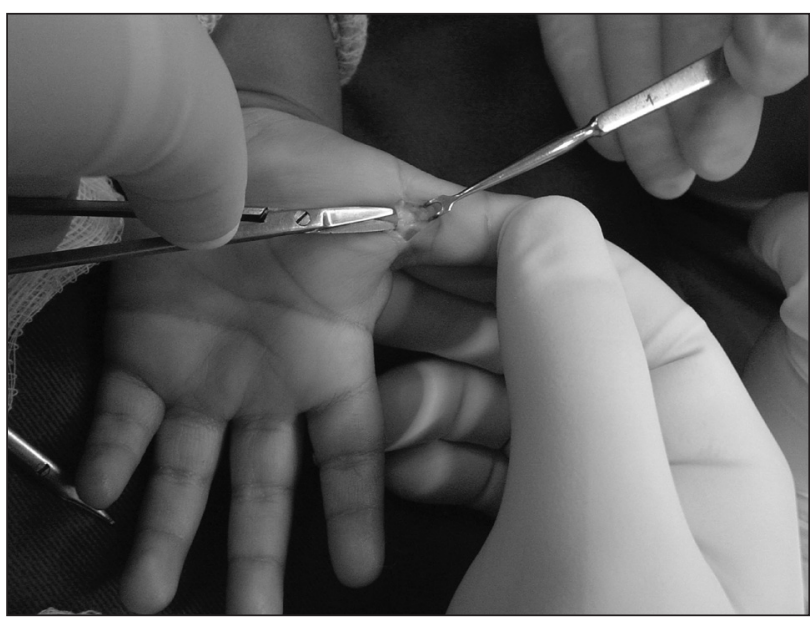

Figura 1 - Espessamento da polia flexora A1.

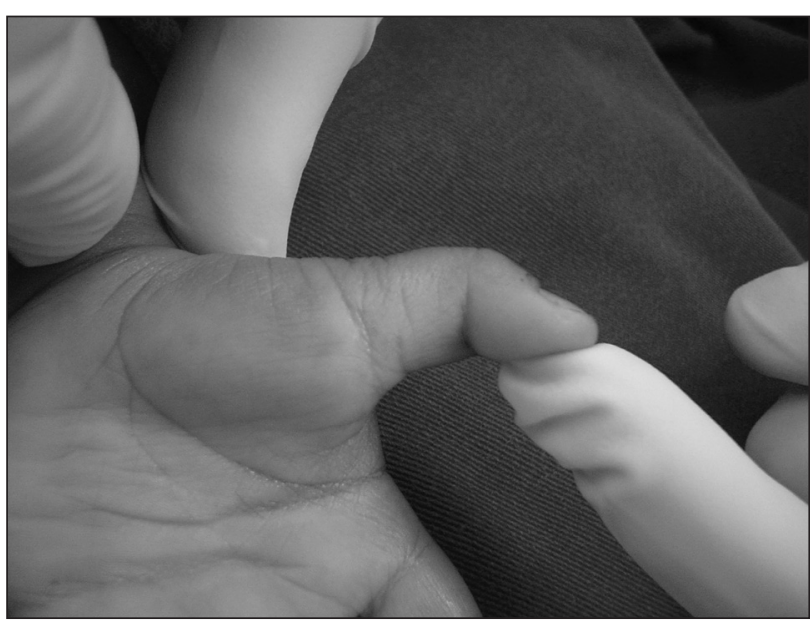

Figura 2 - Dificuldade para extensão do polegar. sentaram com média de três anos, tendo, em alguns casos, já tentado esses tratamentos. Acreditamos ser arriscado o tratamento com liberação percutânea da bainha tendinosa na criança, o qual deve ser evitado, diferentemente no adulto ${ }^{8-10}$.

Observamos a dificuldade de extensão do polegar (Figura 2) e, após a incisão na bainha no tratamento cirúrgico, que foi feita na região da articulação metacarpofalangeana com abordagem transversa na pele. A incisão cutânea longitudinal pode ser feita com menor risco de deiscência da sutura e de secção do nervo digital, mas perde muito para o aspecto estético, porque a incisão cruza a prega palmar transversa. A lesão iatrogênica do nervo digital é descrita como complicação e, felizmente, é rara ${ }^{11}$.

Acreditamos que o uso do torniquete é muito importante para a identificação dos nervos digitais. Sua colocação deve ser muito cuidadosa por tratar-se de crianças e não devemos colocá-lo com muita pressão; assim, o torniquete pneumático pode ser utilizado. Em nossas casuísticas, não o usamos porque achamos mais segura e prática a utilização da faixa de Esmarch, devido às diferenças de tamanho e diâmetro dos membros operados.

Durante as operações, observamos, na maioria dos polegares, abaulamento e intenso espessamento da polia flexora A1; além do aumento do diâmetro do tendão flexor do polegar, que leva a uma desproporção entre conteúdo e continente, o que é típico da tenossinovite estenosante da mão em outros dedos ${ }^{12}$. Acreditamos que não há necessidade de ressecar toda ou parte da polia flexora, a fim de prevenir sua recidiva, pois os resultados em nossa casuística mostraram melhora da extensão do polegar em todos os casos, fato observado já no primeiro retorno ambulatorial.

Não observamos nenhum caso de recidiva. Pelo contrário, existe uma satisfação por parte dos pais, visto que muitos se sentiam negligentes com o diagnóstico tardio. Apesar do cuidado de procurar outras lesões associadas ${ }^{13,14}$, em nossa casuística não foi observada nenhuma alteração local. A utilização do fio de sutura absorvível, poliglecaprone 25 , é prática, pois não requer a retirada dos pontos no ambulatório. Isso porque após três ou quatro semanas a porção do fio que permaneceu interna na pele foi absorvida e os pontos caíram. Acreditamos que os dois polegares que evoluíram com deiscência das suturas e o caso de infecção superficial não ocorreram devido ao fato de o fio ser absorvível.

O uso de cloridrato de bupivacaína $0,5 \%$, sem vasoconstrictor, no local do ato cirúrgico foi realizado em três situações, a pedido dos anestesistas, mas acreditamos não ser necessário, pois atuamos em partes moles; além disso, o procedimento é rápido e não observamos sinais de processo inflamatório local, como hiperemia e hipervascularização local, que necessitasse de analgesia prolongada no período pós-operatório imediato.

Como o tempo cirúrgico médio foi de 27,68 minutos, ou seja, uma cirurgia relativamente rápida, a anestesia inalatória associada ao analgésico não-opioide foi suficiente para garantir o procedimento cirúrgico com muita segurança e dor mínima. Em mãos mais experientes, 
acreditamos que esta média de tempo cirúrgico possa ser diminuída drasticamente. Mesmo assim, chamamos a atenção para a realização da cirurgia com muito cuidado, pois as estruturas são pequenas e todo cuidado é pouco.

Como a anestesia aplicada é geral e inalatória, a internação do paciente é importante, o que oferece segurança para a criança e para a equipe ${ }^{15}$. A utilização do conceito de internação em hospital-dia diminui o custo hospitalar, oferece acompanhamento familiar com conforto e reduz a permanência da criança no hospital. Concluímos que o polegar em gatilho congênito ou tenossinovite estenosante do polegar na criança pode ser tratado com segurança, realizando-se a abertura da polia flexora Al.

\section{Referências}

1. Baek GH, Kim JH, Chung MS, Kang SB, Lee YH, Gong HS. The natural history of pediatric trigger thumb. J Bone Joint Surg Am. 2008;90(5):980-5.

2. Swanson $A B$, Swanson GD, Tada K. A classification for congenital limb malformation. J Hand Surg Am. 1983;8(5 Pt 2):693-702.

3. Moriya K, Uchiyama T, Kawaji Y. Comparison of the surgical outcomes for trigger finger and trigger thumb: preliminary results. Hand Surg. 2005;10(1):83-6.

4. Sampson SP, Badalamente MA, Hurst LC, Seidman J. Pathobiology of the human A1 pulley in trigger finger. J Hand Surg Am. 1991;16(4): 714-21.

5. Lambert MA, Morton RJ, Sloan JP. Controlled study of the use of local steroid injection in the treatment of trigger finger and thumb. J Hand Surg Br. 1992;17(1):69-70.

6. Marks MR, Gunther SF. Efficacy of cortisone injection in treatment of trigger finger and thumbs. J Hand Surg Am. 1989;14:722-7.

7. Newport ML, Lane LB, Stuchin SA. Treatment of trigger finger by steroid injection. J Hand Surg Am. 1990;15(5):748-50.
8. Pope DF, Wolfe SW. Safety and efficacy of percutaneous trigger finger release. J Hand Surg Am. 1995;20(2):280-3.

9. Cohen TJ. Tratamento percutâneo do dedo em gatilho. Rev Bras Ortop. 1996;31(8):690-2.

10. Eastwood DM, Gupta KJ, Johnson DP. Percutaneous release of the trigger finger: an office procedure. J Hand Surg Am. 1992;17(1):114-7.

11. Ogino T. Trigger thumb in children: current recommendations for treatment. J Hand Surg Am. 2008;33(6):982-4.

12. Akhtar S, Bradley MJ, Quinton DN, Burke FD. Management and referral for trigger finger/thumb. BMJ. 2005;331(7507):30-3.

13. Oni 00. A tendon sheath tumor presenting as trigger finger. J Hand Surg Br. 1984;9(3):340.

14. Laing PW. A tendon tumor presenting as a trigger finger. J Hand Surg Br. 1986;11(2):275

15. Bae DS, Sodha S, Waters PM. Surgical treatment of the pediatric trigger finger. J Hand Surg Am. 2007;32(7):1043-7. 